UT-796

\title{
Large Lepton Mixing in a Coset-space Family Unification on $\mathrm{E}_{7} / \mathrm{SU}(5) \times \mathrm{U}(1)^{3}$
}

\author{
J. Sato*and T. Yanagida ${ }^{\dagger}$ \\ Department of Physics, University of Tokyo, \\ Tokyo 113, Japan
}

October 13, 2018

\begin{abstract}
We study a coset-space unification model for families based on $\mathrm{E}_{7} / \mathrm{SU}(5) \times \mathrm{U}(1)^{3}$. We find that qualitative structure of quark and lepton mass matrices in this model describes very well the observation. We stress, in particular, that the large mixing angle, $\sin ^{2} 2 \theta_{\nu_{\mu} \nu_{\tau}} \simeq 1$, required for the atmospheric neutrino oscillation reported by the SuperKamiokande collaboration, is naturally obtained, which is a consequence of unparallel family structure in the present coset-space unification.
\end{abstract}

${ }^{*}$ e-mail address: joe@hep-th.phys.s.u-tokyo.ac.jp

${ }^{\dagger}$ e-mail address: yanagida@hep-th.phys.s.u-tokyo.ac.jp 
The problem of quark-lepton mass matrices is one of the most important issues in particle physics. It is widely expected that these mass matrices contain valuable information on a more fundamental theory beyond the standard model. Much experimental effort has been done to determine the quark masses and mixing angles and as a consequence most of the relevant angles and eigenvalues has been obtained. On the other hand, very little is known for neutrino masses and flavor mixing in the lepton sector. There are only two experimental hints of the flavor mixing at present: one is the well-known solar neutrino deficit [1] and the other the atmospheric neutrino anomaly[2].

A recent report on the atmospheric neutrino from the SuperKamiokande collaboration [3] has presented a convincing evidence that the atmospheric neutrino anomaly is indeed due to neutrino oscillation. They have reported an asymmetry between up- and down-going $\nu_{\mu}$ fluxes. This up-down asymmetry indicates that the $\nu_{\mu}$ 's from overhead do not travel enough to oscillate, while the $\nu_{\mu}$ 's coming from across the earth travel sufficiently to oscillate. Such an asymmetry has not be seen in the $\nu_{e}$ flux. Thus, this implies $\nu_{\mu}-\nu_{\tau}$ oscillation with the mass difference $\delta m_{\nu_{\mu} \nu_{\tau}}^{2} \simeq 10^{-3}-10^{-2} \mathrm{eV}^{2}$ [3] which suggests $m_{\nu_{\tau}} \simeq(0.3-1) \times 10^{-1} \mathrm{eV}$ provided $m_{\nu_{\tau}}>m_{\nu_{\mu}}$.

The above neutrino mass indicates the presence of right-handed neutrinos at the scale $10^{14}-10^{15} \mathrm{GeV}$. 4 . The most natural theory accommodating such right-handed neutrinos is the $\mathrm{SO}(10)$ grand unified theory(GUT). However, the $\mathrm{SO}(10)$ GUT seems to have a difficulty to explain the other surprising fact, i.e. a very large mixing angle $\sin ^{2} 2 \theta_{\nu_{\mu} \nu_{\tau}} \simeq 1[3]$, unless there is a huge hierarchy in Mayorana masses of right-handed neutrinos [5]. An alternative theory accommodating naturally the right-handed neutrinos is the cosetspace family unification [6] in supersymmetric (SUSY) GUT's. Coset-spaces based on $\mathrm{E}_{7}$ are known as unique choices to contain three families of quarks and leptons [7]. Among them $\mathrm{E}_{7} / \mathrm{SU}(5) \times \mathrm{U}(1)^{3}$ is the most interesting, since it contains also three families of right-handed neutrinos as Nambu-Goldstone (NG) multiplets[8].

In this paper we point out that the observed large lepton mixing, $\sin ^{2} 2 \theta_{\nu_{\mu} \nu_{\tau}} \simeq$ 1 , is naturally explained in the $\mathrm{E}_{7} / \mathrm{SU}(5) \times \mathrm{U}(1)^{3}$ model.

The $\mathrm{E}_{7} / \mathrm{SU}(5) \times \mathrm{U}(1)^{3}$ model[7, 8] contains three families of $\mathbf{1 0}_{i}+\mathbf{5}_{i}^{*}+\mathbf{1}_{i}$ $(i=1-3)$ and one 5 as NG multiplets. Here, the $\mathrm{SU}(5)$ is the usual GUT gauge group. Their quantum numbers under the unbroken subgroup are given in Table 1 .

This model can not be quantized in the original form, since there is a nonlinear-sigma model anomaly 9, 8. However, this global obstruction is easily removed [8] by introducing a matter multiplet $5^{*}$ which is also needed for an SU(5) gauge-anomaly cancellation[7]. We assume that some explicit breaking induces an invariant mass for the NG $\mathbf{5}$ and this matter $\mathbf{5}^{*}$ and we neglect them in our discussion.

In addition to the NG multiplets we introduce a pair of Higgs multiplets 


\begin{tabular}{c|ccc}
$\mathrm{SU}(5)$ & $\mathrm{U}(1)_{1}$ & $\mathrm{U}(1)_{2}$ & $\mathrm{U}(1)_{3}$ \\
\hline $\mathbf{1 0}_{1}$ & 0 & 0 & 4 \\
$\mathbf{1 0}_{2}$ & 0 & 3 & -1 \\
$\mathbf{1 0}_{3}$ & 2 & -1 & -1 \\
$\mathbf{5}_{1}^{*}$ & 0 & 3 & 3 \\
$\mathbf{5}_{2}^{*}$ & 2 & -1 & 3 \\
$\mathbf{5}_{3}^{*}$ & 2 & 2 & -2 \\
$\mathbf{1}_{1}$ & 0 & 3 & -5 \\
$\mathbf{1}_{2}$ & 2 & -1 & -5 \\
$\mathbf{1}_{3}$ & 2 & -4 & 0 \\
\hline $\mathbf{5}$ & 2 & 2 & 2
\end{tabular}

Table 1: $\mathrm{U}(1)$ charges of the $\mathrm{NG}$ multiplets. The $\mathrm{U}(1)_{1}, \mathrm{U}(1)_{2}$ and $\mathrm{U}(1)_{3}$ are the unbroken $\mathrm{U}(1)$ 's of coset-subspaces $\mathrm{E}_{7} / \mathrm{E}_{6} \times \mathrm{U}(1), \mathrm{E}_{6} / \mathrm{SO}(10) \times \mathrm{U}(1)$ and $\mathrm{SO}(10) / \mathrm{SU}(5) \times \mathrm{U}(1)$, respectively.

$\mathbf{5}_{H}$ and $\mathbf{5}_{H}^{*}$. As long as the global $\mathrm{E}_{7}$ is exact these Higgs multiplets never have Yukawa couplings to the NG quarks and leptons. Thus, the observed hierarchy in quark-lepton mass matrices is regarded as a consequence of a hierarchy in the explicit breaking of the global $E_{7}$. This situation is very similar to that in the QCD, where the mass hierarchy between NG pions and kaons $\left(m_{K}^{2} \gg m_{\pi}^{2}\right)$ is originated from the hierarchy in quark masses $\left(m_{s} \gg\right.$ $\left.m_{u, d}\right)$ which are explicit breaking parameters of the chiral $\mathrm{SU}(3)_{L} \times \mathrm{SU}(3)_{R}$.

We consider three steps for the explicit breaking:

$$
\mathrm{E}_{7} \underset{\epsilon_{0}}{\longrightarrow} \mathrm{E}_{6} \underset{\epsilon_{1}}{\longrightarrow} \mathrm{SO}(10) \underset{\epsilon_{2}}{\longrightarrow} \mathrm{SU}(5)
$$

which leads to the mass hierarchy

$$
\begin{gathered}
m_{t} \gg m_{c} \gg m_{u} \\
m_{b} \gg m_{s} \gg m_{d} \\
m_{\tau} \gg m_{\mu} \gg m_{e} .
\end{gathered}
$$

To realize this hierarchy we assume that the global $\mathrm{E}_{7}$ is broken explicitly by the fundamental representation of $\mathrm{E}_{7}, \mathbf{5 6}$, which contains six breaking parameters, $\epsilon_{0}, \bar{\epsilon}_{0}, \epsilon_{1}, \bar{\epsilon}_{1}, \epsilon_{2}, \bar{\epsilon}_{2}$ that are all singlets of $\mathrm{SU}(5)$. They carry $\mathrm{U}(1)$ charges as

$$
\begin{array}{rr}
\epsilon_{0}(-3,0,0), & \bar{\epsilon}_{0}(3,0,0) \\
\epsilon_{1}(-1,-4,0), & \bar{\epsilon}_{1}(1,4,0) \\
\epsilon_{2}(-1,-1,-5), & \bar{\epsilon}_{2}(1,1,5)
\end{array}
$$


where the numbers in each parenthesis denote charges of $\mathrm{U}(1)_{1} \times \mathrm{U}(1)_{2} \times \mathrm{U}(1)_{3}$. The desired hierarchy in eq.(1) is represented by

$$
\epsilon_{0} \gg \epsilon_{1} \gg \epsilon_{2}
$$

The structure of Yukawa couplings for the NG quarks and leptons depends on $\mathrm{U}(1)$ charges of the Higgs $\mathbf{5}_{H}$ and $\mathbf{5}_{H}^{*}$. To determine them, we consider that the Higgs multiplets $\mathbf{5}_{H}$ and $\mathbf{5}_{H}^{*}$ belong to $\mathbf{2 7}$ of $\mathrm{E}_{6}$ in $\mathbf{1 3 3}$ of $\mathrm{E}_{7}$. Then, $\mathrm{U}(1)$ charges for the $\boldsymbol{5}_{H}$ are given by

$$
\mathbf{5}_{H}(2,2,2) \text {. }
$$

The Higgs $5_{H}^{*}$ is a linear combination of two $5^{*}$ 's in 27 of $\mathrm{E}_{6}$ 円 as

$$
\mathbf{5}_{H}^{*}=\sin \theta \mathbf{5}_{16}^{*}+\cos \theta \mathbf{5}_{10}^{*}
$$

where $\mathrm{U}(1)$ charges for $\mathbf{5}_{16}^{*}$ and $\mathbf{5}_{10}^{*}$ are given by ${ }^{2}$

$$
\mathbf{5}_{16}^{*}(2,-1,3) \text { and } \mathbf{5}_{10}^{*}(2,2,-2) \text {. }
$$

We now discuss Yukawa couplings for the quark and lepton multiplets. In general, Yukawa couplings are given in a form $a_{n} \epsilon^{n} \psi \psi H$ where $\epsilon, \psi$ and $H$ stand for the explicit breaking parameters, the NG multiplets and the Higgs multiplets, respectively. By our choice of the $U(1)$ charges for the explicit breaking parameters and Higgs multiplets, Yukawa couplings take the following form in the leading order of the explicit breaking parameters, $\epsilon$ 's;

$$
\begin{aligned}
W & =W_{U}+W_{D}+W_{E}+W_{\nu}, \\
W_{U} & =\sum_{i j} a_{i j} Y_{U i j} \mathbf{1 0}_{i} \mathbf{1 0}_{j} \mathbf{5}_{H}, \\
W_{D} & =W_{E}=\sum_{i j} b_{i j} Y_{D / E i j} \boldsymbol{5}_{i}^{*} \mathbf{1 0}_{j} \mathbf{5}_{H}^{*}, \\
W_{\nu} & =\sum_{i j} c_{i j} Y_{\nu i j} \mathbf{5}_{i}^{*} \mathbf{1}_{j} \boldsymbol{5}_{H},
\end{aligned}
$$

where $W_{U}, W_{D}, W_{E}$ and $W_{\nu}$ represent superpotentials of Yukawa couplings for up-type quarks, down-type quarks, charged leptons and neutrinos. In

$1 \mathbf{2 7}$ of $\mathrm{E}_{6}$ is decomposed to $\mathbf{1 6}+\mathbf{1 0}+\mathbf{1}$ of $\mathrm{SO}(10)$. The $\mathbf{1 6}$ and $\mathbf{1 0}$ contain one $\mathbf{5}$ and two $5^{*}$ of $\mathrm{SU}(5)$.

2 The orthogonal combination of the $\mathbf{5}_{16}^{*}$ and $\mathbf{5}_{10}^{*}$ is assumed to have a GUT scale mass. We also assume that color triplets in $\mathbf{5}_{H}$ and $\boldsymbol{5}_{H}^{*}$ receive a GUT scale mass after the spontaneous breakdown of the SU(5) GUT. This requires a fine tuning. We do not, however, discuss this fine tuning problem here, since it is beyond the scope of this paper. 


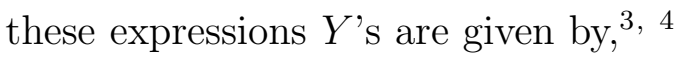

$$
\begin{gathered}
Y_{U} \simeq\left(\begin{array}{ccc}
\epsilon_{2}^{2} & \epsilon_{1} \epsilon_{2} & \epsilon_{0} \epsilon_{2} \\
\epsilon_{1} \epsilon_{2} & \epsilon_{1}^{2} & \epsilon_{0} \epsilon_{1} \\
\epsilon_{0} \epsilon_{2} & \epsilon_{0} \epsilon_{1} & \epsilon_{0}^{2}
\end{array}\right), \\
Y_{D / E} \simeq\left(\begin{array}{ccc}
\epsilon_{1} \epsilon_{2} \cos \theta & \epsilon_{1}^{2} \cos \theta & \epsilon_{0} \epsilon_{1} \cos \theta \\
\epsilon_{0} \epsilon_{2} \cos \theta & \epsilon_{0} \epsilon_{1} \cos \theta & \epsilon_{0}^{2} \cos \theta \\
\epsilon_{0} \epsilon_{2} \sin \theta & \epsilon_{0} \epsilon_{1} \sin \theta & \epsilon_{0}^{2} \sin \theta
\end{array}\right), \\
Y_{\nu} \simeq\left(\begin{array}{ccc}
\epsilon_{1}^{2} & \epsilon_{0} \epsilon_{1} & \epsilon_{0} \epsilon_{2} \\
\epsilon_{0} \epsilon_{1} & \epsilon_{0}^{2} & 0 \\
0 & 0 & \epsilon_{0}^{2}
\end{array}\right)
\end{gathered}
$$

We have assumed the $\mathrm{E}_{7}$ representations for $\epsilon_{i}, \mathbf{5}_{H}$ and $\boldsymbol{5}_{H}^{*}$ to determine their $\mathrm{U}(1)$ charges. However, we consider that this assumption is over statement since the $\mathrm{E}_{7}$ is already spontaneously broken. What is relevant to our analysis is only their charges of the unbroken subgroup $\mathrm{SU}(5) \times \mathrm{U}(1)^{3}$. With this general consideration it is impossible to estimate the coefficients $a_{i j}, b_{i j}$ and $c_{i j}$ in eqs.(9), (10) and (11) and hence we assume that they are of $\mathrm{O}(1)$ in this paper.

From the above Yukawa couplings in eqs.(12) and (13) we easily derive the following mass relations;

$$
\begin{array}{rlrl}
\frac{m_{u}}{m_{c}} & \sim \frac{\epsilon_{2}^{2}}{\epsilon_{1}^{2}}, & \frac{m_{c}}{m_{t}} & \sim \frac{\epsilon_{1}^{2}}{\epsilon_{0}^{2}} \\
\frac{m_{e}}{m_{\mu}}=\frac{m_{d}}{m_{s}} & \sim \frac{\epsilon_{2}}{\epsilon_{0}} \sin ^{-1} \theta, & \frac{m_{\mu}}{m_{\tau}}=\frac{m_{s}}{m_{b}} \sim \frac{\epsilon_{1}}{\epsilon_{0}} \sin \theta \cos \theta .
\end{array}
$$

These relations describe very well the observed mass relations provided that

$$
\frac{\epsilon_{1}}{\epsilon_{0}} \sim 0.1, \frac{\epsilon_{2}}{\epsilon_{0}} \sim 0.01 \text { and } \tan \theta \sim 1
$$

We see that the Cabibbo-Kobayashi-Maskawa mixing angles for quarks between the 1st and the 2nd, the 2nd and the 3rd, and the 3rd and the 1st family are of the order $\epsilon_{2} / \epsilon_{1}, \epsilon_{1} / \epsilon_{0}$, and $\epsilon_{2} / \epsilon_{0}$, respectively. It also describes

\footnotetext{
${ }^{3}$ One may wonder that in eq.(13) the $(3,1)$ element of $Y_{D / E}$, has a term of $\epsilon_{0} \epsilon_{1}$. We do not think that such a term appears there, since in the limit $\epsilon_{2} \rightarrow 0$, the global SO(10) symmetry becomes exact and the $\mathbf{1 0}_{1}$ is the true NG multiplet which has no Yukawa interaction in the superpotential.

${ }^{4}$ Precisely speaking, our coset-space $\mathrm{E}_{7} / \mathrm{SU}(5) \times \mathrm{U}(1)^{3}$ contains three dimensional parameters $f_{0}, f_{1}$ and $f_{2}$. We assume $f_{0} \sim f_{1} \sim f_{2}$ here, for simplicity. However, even if it is not the case, one obtains the same form of Yukawa couplings as in eqs.(12), (13) and (14) by redefining $\epsilon$ 's as $\epsilon_{i}=\tilde{\epsilon}_{i} / f_{i}(i=0,1,2)$ where $\tilde{\epsilon}_{i}$ are original dimensional parameters for the explicit $\mathrm{E}_{7}$ breakings.
} 
the observed mixing angles very well provided that the relations in eq.(16) are satisfied.

We do not further mention details of the mass relations since there should be corrections to the mass matrices in eqs.(12) and (13) from some higher dimensional operators which may affect masses for lighter particles significantly. Otherwise, we have a $\mathrm{SU}(5) \mathrm{GUT}$ relation, $m_{d}=m_{e}$, which seems unrealistic 10.

So far, we have discussed the mass matrices for quarks and charged leptons and found that the qualitative global structure of the obtained matrices fits very well the observed mass spectrum for quarks and charged leptons (except for $m_{d}=m_{e}$ ) and mixing angles for quarks if the relations in eq.(16) are satisfied?.

We are now at the point to discuss neutrino masses and lepton mixings. We assume that Mayorana masses for right-handed neutrinos $N_{i}$ are induced by $\mathrm{SU}(5)$ singlet Higgs multiplets $\bar{s}_{i}(\mathbf{1})$. We introduce two singlets $\overline{s_{1}}(\mathbf{1})$ and $\bar{s}_{2}(\mathbf{1})$ whose $\mathrm{U}(1)$ charges 5 are given by

$$
\bar{s}_{1}(1,4,0) \text { and } \bar{s}_{2}(1,1,5) \text {. }
$$

Their vacuum expectation values, $\left\langle\overline{s_{1}}\right\rangle$ and $\left\langle\overline{s_{2}}\right\rangle$ are expected to be of order of the $\mathrm{SU}(5) \mathrm{GUT}$ scale $\sim 10^{16} \mathrm{GeV}$.

Majorana masses for $N_{i}$ are induced from nonrenormalizable interactions of a form;"]

$$
W_{N}=\frac{\epsilon^{2}}{M_{G}} N_{i} N_{j} \bar{s}_{k} \bar{s}_{l} .
$$

Here, $M_{G}$ is the gravitational scale $M_{G} \simeq 2.4 \times 10^{18} \mathrm{GeV}$. Then, the matrix of the Majorana masses takes the following form;

$$
M_{\nu_{R}}=\frac{1}{M_{G}}\left(\begin{array}{ccc}
\epsilon_{1}^{2} \overline{s_{2}} & \epsilon_{0} \epsilon_{1}{\overline{s_{2}}}^{2} & \epsilon_{0} \epsilon_{1} \overline{s_{1}} \overline{s_{2}} \\
\epsilon_{0} \epsilon_{1} \overline{s_{2}} & \epsilon_{0}^{2} \overline{s_{2}} & \epsilon_{0}^{2} \overline{s_{1}} \overline{s_{2}} \\
\epsilon_{0} \epsilon_{1} \overline{s_{1}} \overline{s_{2}} & \epsilon_{0}^{2} \overline{s_{1}} \overline{s_{2}} & \epsilon_{0}^{2} \overline{s_{1}}
\end{array}\right),
$$

where all elements are multiplied by undetermined factors of $\mathrm{O}(1)$ like in the case for quarks and leptons.

The neutrino masses are given by

$$
m_{\nu} \simeq m_{\nu_{D}} M_{\nu_{R}}^{-1} m_{\nu_{D}}^{T}
$$

where

$$
\left(m_{\nu_{D}}\right)_{i j}=c_{i j} Y_{\nu i j}\left\langle\mathbf{5}_{H}\right\rangle .
$$

\footnotetext{
${ }^{5}$ The observed mass for the strange quark seems somewhat smaller than the $\mathrm{SU}(5)$ GUT value 10 .

6 These $\overline{s_{i}}(\mathbf{1})$ are regarded as $\mathrm{SU}(5)$ singlet components of $\mathbf{5 6}$ of $\mathrm{E}_{7}$.

${ }^{7}$ Other mass terms such as $\epsilon^{2} N_{i} N_{j}$ can be forbidden by some chiral symmetry.

${ }^{8}$ The mass term of the form $\epsilon^{4} N_{i} N_{j}$ may produce a similar form to eq.(19) if $\bar{\epsilon}_{0}=0$ and $\bar{\epsilon}_{1}, \bar{\epsilon}_{2} \neq 0$.
} 
Three eigenvalues of the matrix in eq.(20) are of order, $m_{\nu_{1}} \sim \epsilon_{1}^{2} M_{G}\left\langle\mathbf{5}_{H}\right\rangle^{2} /\left\langle\bar{s}_{2}\right\rangle^{2}$, $m_{\nu_{2}} \sim \epsilon_{0}^{2} M_{G}\left\langle\mathbf{5}_{H}\right\rangle^{2} /\left\langle\bar{s}_{2}\right\rangle^{2}$ and $m_{\nu_{3}} \sim \epsilon_{0}^{2} M_{G}\left\langle\mathbf{5}_{H}\right\rangle^{2} /\left\langle\bar{s}_{1}\right\rangle^{2}$. It is remarkable that for $\left\langle\mathbf{5}_{H}\right\rangle \sim 100 \mathrm{GeV}, \epsilon_{0} \sim 1$ and $\left\langle\bar{s}_{i}\right\rangle \sim 10^{16} \mathrm{GeV}$ we get the desired mass for neutrino $m_{\nu_{i}} \sim 0.1 \mathrm{eV}$.

From the Mikheev-Smirnov-Wolfenstein solution(MSW) [1] to the solar neutrino problem, we have 12, 13]

$$
\delta m_{\nu_{e} \nu_{\mu}}^{2} \simeq 10^{-6}-10^{-5} \mathrm{eV}^{2} .
$$

We see that there are two choices

$$
\left(\frac{\left\langle\bar{s}_{1}\right\rangle}{\left\langle\bar{s}_{2}\right\rangle}\right)^{2} \sim 10^{-2}-10^{-1} \text { or }\left(\frac{\left\langle\bar{s}_{2}\right\rangle}{\left\langle\bar{s}_{1}\right\rangle}\right)^{2} \sim 10^{-2}-10^{-1}
$$

to account for atmospheric and solar neutrino anomalies, simultaneously. Thus, all off-diagonal elements of the diagonalization matrix for the neutrino mass matrix in eq.(20) are of $\mathrm{O}(0.1)$ in either cases.

However, it is very interesting that the mixing angle for lepton doublets which mixes charged leptons in the second and the third family is of order $\tan \theta$ (see eq.(13)) and hence of the order 1 . This means, together with the above result, that the weak mixing angle relevant for $\nu_{\mu}-\nu_{\tau}$ oscillation can be so large, $\sin ^{2} 2 \theta_{\nu_{\mu} \nu_{\tau}} \simeq 1$, as required for explaining the observed atmospheric neutrino anomaly. On the other hand, the mixing angle for $\nu_{\mu}-\nu_{e}$ oscillation is very small, $\theta_{\nu_{\mu} \nu_{e}} \sim \mathrm{O}(0.1)$, which may fit the small angle MSW solution [12, 13] to the solar neutrino problem.

In this paper we have shown that the coset-space family unification on $\mathrm{E}_{7} / \mathrm{SU}(5) \times \mathrm{U}(1)^{3}$ naturally accommodates the large lepton mixing, $\sin ^{2} 2 \theta_{\nu_{\mu} \nu_{\tau}} \simeq$

1 , necessary for explaining the atmospheric neutrino anomaly reported by the SuperKamiokande collaboration [3]. The main reason why we have a large mixing of the $\mathrm{SU}(2)$ lepton doublets in the second and the third family is the twisted structure of family. Namely, the $\mathbf{5}^{*}$ s in the second and the third family both live on the same coset-subspace $\mathrm{E}_{7} / \mathrm{E}_{6} \times \mathrm{U}(1)$. On the other hand the 10's in the third, the second and the first family live on the separate coset-subspaces, $\mathrm{E}_{7} / \mathrm{E}_{6} \times \mathrm{U}(1), \mathrm{E}_{6} / \mathrm{SO}(10) \times \mathrm{U}(1)$ and $\mathrm{SO}(10) / \mathrm{SU}(5) \times \mathrm{U}(1)$, respectively. This unparallel family structure is an unique feature of the present coset-space family unification.

\section{References}

[1] Homestake Collaboration, B. T. Cleveland et al., Nucl. Phys. B (Proc. Suppl.) 38, 47 (1995);

Kamiokande Collaboration, Y. Suzuki, Nucl. Phys. B (Proc. Suppl.) 38,54 (1995);

GALLEX Collaboration, P. Anselmann et al., Phys. Lett. B 357, 237 
(1995);

SAGE Collaboration, J. N. Abdurashitov et al., Phys. Lett. B 328, 234 (1994).

[2] Kamiokande Collaboration, K.S. Hirata et al., Phys. Lett. B 205, 416 (1988); ibid. B 280, 146 (1992) ; Y. Fukuda et al., Phys. Lett. B 335, 237 (1994);

IMB Collaboration, D. Casper et al., Phys. Rev. Lett. 66, 2561 (1991);

R. Becker-Szendy et al., Phys. Rev. D 46, 3720 (1992);

SOUDAN2 Collaboration, T. Kafka, Nucl. Phys. B (Proc. Suppl.) 35, 427 (1994); M. C. Goodman, ibid. 38 (1995) 337; W. W. M. Allison et al., Phys. Lett. B 391, 491 (1997).

[3] Y. Totsuka, invited talk at the 18th International Symposium on LeptonPhoton Interaction, July 28 - August 1, 1997 Hamburg.

[4] T. Yanagida, in Proceedings of the Workshop on Unified Theory and Baryon Number in the Universe, edited by A. Sawada and H. Sugawara, (KEK, Thukuba, Japan, 1979);

M. Gell-Mann, P. Ramond and R. Slansky, in Supergravity, edited by F. van Nieuwenhuizen and D. Freedman, (North Holland, 1979).

[5] T. Yanagida and M. Yoshimura, Phys. Lett. B97, 99 (1980); G. Branco and A. Masiero, Phys. Lett. B97, 95 (1980).

[6] W. Buchmuller, R. D. Peccei and T. Yanagida, Nucl. Phys. B 227, 503 (1983).

[7] T. Kugo and T. Yanagida, Phys. Lett. B 134, 313 (1984).

[8] T. Yanagida and Y. Yasui, Nucl. Phys. B 269, 576 (1986).

[9] G. Moore and P. Nelson, Phys. Rev. Lett. 53, 1519 (1984); Commun. Math Phys. 100, 83 (1985);

P. di Vecchia, S. Ferrara and L. Girardello, Phys. Lett. B 151, 199 (1985);

E. Cohen and C. Gomez, Nucl. Phys. B 254, 235 (1985).

[10] Particle Data Group, Phys. Rev. D 54, 1 (1996).

[11] L. Wolfenstein, Phys. Rev. D 17, 2369 (1978);

S. P. Mikheev and A. Yu. Smirnov, Sov. J. Nucl. Phys. 42, 913 (1985).

[12] J. N. Bahcall and P. I. Krastev, Phys. Rev. D 53,4211 (1996);

G. L. Fogli, E. Lisi and D. Montanino, hep-ph/9709473.

[13] See, for a review, M. Fukugita and T. Yanagida, in Physics and Astrophysics of Neutrinos, edited by M. Fukugita and A. Suzuki (SpringerVerlag, Tokyo, 1994). 